\title{
Fetal Transcerebellar Diameter to Abdominal Circumference Ratio (TCD/AC) in the Assessment of Normal Fetal Growth
}

\author{
${ }^{1}$ Hema Dhumale, ${ }^{1}$ Yeshita V Pujar, ${ }^{2}$ Jyotsana C Shravage, ${ }^{2} \mathrm{MB}$ Bellad \\ ${ }^{1}$ Bhavana Y Sherigar, ${ }^{1}$ Geeta S Durdi, ${ }^{3}$ Sheetal S Amber \\ ${ }^{1}$ Associate Professor, Department of Obstetrics and Gynecology, Jawaharlal Nehru Medical College, Nehru Nagar, Belgaum, Karnataka, India \\ ${ }^{2}$ Professor, Department of Obstetrics and Gynecology, Jawaharlal Nehru Medical College, Nehru Nagar, Belgaum, Karnataka, India \\ ${ }^{3}$ Assistant Professor, Department of Obstetrics and Gynecology, Jawaharlal Nehru Medical College, Nehru Nagar, Belgaum, Karnataka, India
}

Correspondence: Hema Dhumale, Associate Professor, Department of Obstetrics and Gynecology, Jawaharlal Nehru Medical College, Nehru Nagar, Belgaum-590 010, Karnataka, India, Phone: 9480537499, e-mail: drhemadhumale@gmail.com

\section{ABSTRACT}

Objective: To derive population specific transverse cerebellar diameter/abdominal circumference ratio (TCD/AC ratio) for the assessment of normal fetal growth.

Materials and methods: A one-year prospective observational study conducted at the KLE University's teaching hospital, Belgaum. The TCD/AC ratio was calculated for 434 obstetric patients, who met the inclusion criteria and the arithmetic mean and 1 standard deviation (SD) was calculated for the population studied.

Results: Within the study population, the TCD/AC ratio assessed for normal fetal growth with gestational age ranging from 18 to 34 weeks was found to be a constant with a mean of $13.56+/-1.21$ (1 SD).

Conclusion: A normal standard value to assess normal fetal growth is required for a given set of population in order to be compared with those fetuses with intrauterine growth restriction (IUGR). The TCD/AC value in our study remained a constant between gestational ages ranging from 18 to 34 weeks with a mean of $13.56+/-1.21$.

Keywords: Transverse cerebellar diameter/Abdominal circumference ratio (TCD/AC ratio), Ultrasonography, Fetal growth, Intrauterine growth restriction.

\section{INTRODUCTION}

Many fetal parameters can be measured by ultrasonography and have been correlated with gestational age. ${ }^{1}$ Ultrasonography has become the principal means by which fetal well being is monitored and deviant fetal growth is detected. ${ }^{2}$ The biometric parameters used most frequently to evaluate fetal growth include the fetal biparietal diameter (BPD), head circumference (HC), abdominal circumference (AC), and femur length (FL). Precise knowledge of gestational age is required to accurately utilize these biometric parameters to assess the fetal growth. ${ }^{2}$ A major limitation of most of these parameters in diagnosing abnormal fetal growth is their dependence on accurate knowledge of the gestational age. $^{2}$

Therefore, the usefulness of these biometric parameters in establishing gestational age in the third trimester of pregnancy or in assessing fetal growth in pregnancies with uncertain dating is limited. ${ }^{3}$ In most of such cases, serial ultrasonographic evaluation is required to differentiate normal from abnormal fetal growth, which may delay the diagnosis and appropriate intervention. ${ }^{1}$

Hence, there is a need for gestational age independent parameter to assess fetal growth. The femur length/abdominal circumference (FL/AC ratio) and transcerebellar/abdominal circumference (TCD/AC ratio) have been found to be constant and gestational age independent parameters to assess fetal growth. $^{2}$

Though FL/AC ratio is a useful measure of asymmetric growth restriction, it is less sensitive in detecting intrauterine growth restriction (IUGR) and there is considerable overlap of FL/AC ratio in normal and growth restricted fetuses without a single cut off value to identify IUGR. ${ }^{4,5}$ Moreover, FL/AC ratio is not useful in symmetrical IUGR because both the parameters are affected. ${ }^{5}$

The transverse cerebellar diameter (TCD) is wellestablished in the ultrasound literature as a reliable parameter for estimating gestational age. ${ }^{7}$ Measurements of fetal TCD have been shown to correlate with gestational age. The TCD/AC ratio is a constant between 14 and 42 weeks and, therefore is a gestational age independent factor to assess the fetal growth. ${ }^{1}$ While all fetal biometric parameters are affected by intrauterine growth restriction, the effect on the size of the cerebellum is minimal. ${ }^{2,8}$ Hence, deviation of TCD/AC ratio is a sensitive indicator of IUGR. The differences in the TCD/AC ratios and their sensitivities in detecting IUGR in different studies could 
be due to different population types studied. ${ }^{3}$ As the biometric parameters change due to genetic and racial factors, there is a need to either develop population specific nomograms or to prospectively revalidate one of the chosen nomograms for appropriate clinical use. ${ }^{9}$

\section{MATERIALS AND METHODS}

This was a prospective observational study conducted in the Department of Obstetrics and Gynecology at KLE University's Teaching Hospital, Belgaum, Karnataka, India for a period of one year from September 2008 to October 2009. All antenatal women undergoing Obstetric ultrasound between 18-34 weeks of singleton pregnancy and confirmed gestational age (regular menstrual history with known last menstrual period and/or Ultrasound confirmation of gestational age by means of crown rump length measurement) were included in the study. IUGR, macrosomia, hypertension, diabetes mellitus, cardiac diseases, fetal congenital abnormalities and failure to clearly visualize/ measure the fetal cerebellum were excluded from the study. A total of 434 antenatal women were eligible for the study.

Ultrasound examination was carried out using Philips HD11 ultrasound machine with a convex probe of $3.5 \mathrm{MHz}$ by two trained obstetric sonologists. Fetal biometric parameters like biparietal diameter, head circumference, transverse cerebellar diameter, abdominal circumference and femur length were measured. The cerebellum was located in the posterior fossa and visualized by a slight posterior and inferior rotation of the transducer at the level of the BPD. Electronic calipers was used to measure the TCD in an outer to outer fashion as described by Goldstein et $\mathrm{al}^{3}$ (Fig. 1). The AC was measured from a transaxial view of the abdomen at the level of the junction of the umbilical vein with the left portal vein. The TCD/AC ratio was calculated by dividing the TCD by the AC and then multiplying by $100 .^{2}$

\section{RESULTS}

The mean TCD/AC ratio at different gestational age was calculated and found to be constant in our study, which included

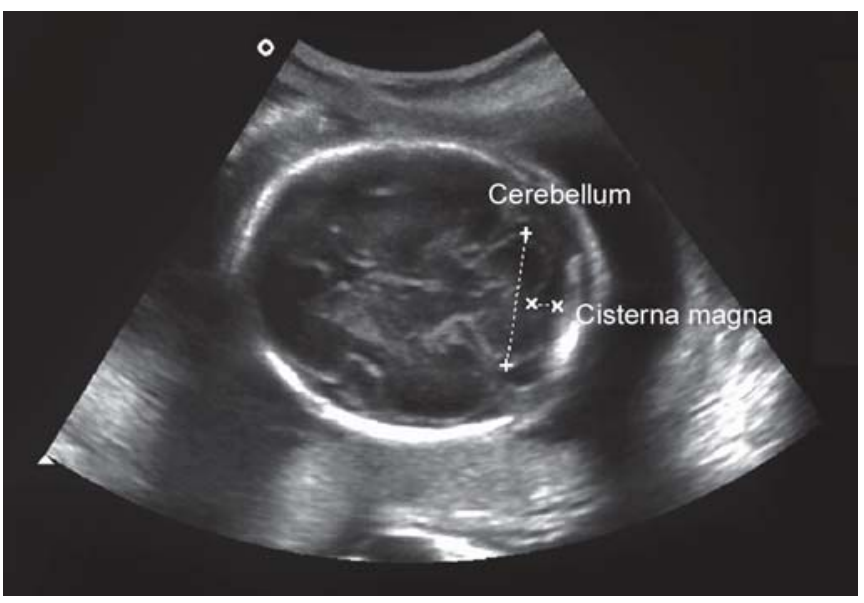

Fig. 1: Measurement of transcerebellar diameter
Table 1: The mean TCD/AC ratio at different periods of gestation

\begin{tabular}{lc}
\hline Period of gestation & TCD/AC ratio (Mean) \\
\hline - 18 to 22 weeks (N-234) & 13.35 \\
- 23 to 26 weeks (N-108) & 13.45 \\
- 27 to 30 weeks (N-77) & 13.55 \\
- 31 to 34 weeks (N-15) & 14.0 \\
\hline
\end{tabular}

Table 2: Arithmetic mean and standard deviation values

\begin{tabular}{ll}
\hline Parameters & Result \\
\hline - Mean TCD/AC ratio & 13.56 \\
- Standard deviation & 1.21 \\
\hline
\end{tabular}

patients between 18 and 34 weeks period of gestation (Table 1). The arithmetic mean and standard deviation was calculated for the sample size of 434 cases (Table 2).

\section{DISCUSSION}

It has been observed that cortical blood flow is decreased in hypoxic fetus but cerebellar blood flow remains unchanged. Therefore, TCD/AC ratio is a good parameter for detecting IUGR in cases of uncertain dates because it utilizes parameters like abdominal circumference, which is the most sensitive parameter of fetal growth and TCD which is not affected or minimally affected from growth restriction resulting in an increased ratio reflecting IUGR regardless of gestational age. ${ }^{2}$ Therefore, TCD/AC ratio has the advantage of being not only a relatively good predictor of IUGR but also a gestational age independent parameter. ${ }^{7}$ Literature suggests TCD/AC ratio 2 $\mathrm{SD}$ above the mean is a sensitive predictor of IUGR. ${ }^{3}$ The TCD/AC ratio was found to be gestational age independent in our study between 18 and 34 weeks of gestation and the population specific TCD/AC ratio (mean +/- SD) was 13.56 with standard deviation of 1.21 for normal fetal growth. The $\mathrm{TCD} / \mathrm{AC}$ ratio greater than $2 \mathrm{SD}$ of the mean, i.e. greater than $13.56+(2 \times 1.21)=15.98$ would suggest IUGR in our population. A study done on 829 low risk obstetric patients concluded that TCD/AC ratio was gestational age independent between 14 and 42 weeks with a mean of $13.68+/-0.96$. Ratio exceeding 2SD of the mean was significantly associated with birth of a small for gestational age infant being abnormal in $98 \%$ and $71 \%$ of asymmetrically and symmetrically growth restricted fetuses, respectively. ${ }^{2}$ Two earlier studies, which studied the usefulness of TCD/AC ratio in IUGR fetuses, concluded that the TCD/AC ratio cut off value suggestive of IUGR was 15.4 and 15.9. ${ }^{6,8}$ The comparison of our study results with the studies conducted earlier showed that the TCD/AC ratio is a gestational age independent constant parameter further 
the mean TCD/AC ratio for normal fetal growth and the 2SD or cut off values for diagnosis of IUGR are similar between our study and the earlier studies. ${ }^{2,8}$

This study will help us to utilize our population specific TCD/AC ratio to detect IUGR particularly in women, who present in the third trimester of pregnancy with unknown dates or no previous antenatal ultrasonography reports.

\section{CONCLUSION}

The population specific TCD/AC ratio reference value for normal fetal growth for our institution was found to be 13.56 with standard deviation value of 1.21. A TCD/AC ratio greater than 2 SD of the mean, i.e. greater than 15.98, is considered suspicious and suggestive of fetal growth restriction. After having established our population specific TCD/AC ratio for assessing normal fetal growth, this study will help us to further utilize this ratio to detect IUGR in the same population.

\section{REFERENCES}

1. Vinkesteijn ASM. Muder PGH, Wladimiroff JW. Fetal transverse cerebellar diameter measurements in normal and reduced fetal growth. Ultrasound Obstet Gynecol 2000;15: 47-51.

2. Meyer WJ, Gauthier D, Ramakrishnan V, Sipos J. Ultrasonographic detection of abnormal fetal growth with the gestational age: Independent transverse cerebellar diameter to abdominal circmference ratio. Am J Obstet Gynecol 1994;171:1057-63.

3. Haller H, Petrovic O, Rukavina B. Fetal transverse cerebellar diameter/abdominal circumference ratio in assessing fetal size. Int J Gynec Obstet 1995;50:159-63.

4. Hadlock FP, Deter RL, Harrist RB, Roecker E, Park SK. A dateindependent of intrauterine growth retardation: Femur length/ abdominal circumference ratio. Am J Roentgenol 1983;141(5):979-84.

5. Benson CB, Doubilet PM, Saltzman DH, Jones TB. FL/AC ratio: Poor predictor of intrauterine growth retardation. J Ultrsound Med Mar 1986;5(3):141-44.

6. Tongsong T, Wanapirak C, Thongpadungroj T. Sonographic diagnosis of intrauterine growth restriction (IUGR) by fetal transverse cerebellar diameter (TCD)/abdominal circumference (AC) ratio. Int J Gynecol Obstet 1999;66:1-5.

7. Chavez MR, Ananth CV, Smulian JC, Lashley S, Kontopoulos EV, Anthony M, Vintzileos AM. Fetal transcerebellar diameter nomogram in singleton gestations with special emphasis in the third trimester: A comparison with previously published nomograms. Am J Obstet Gynecol 2003;189(4):1021-25.

8. Campbell WA, Vintzileos AM, Rodis JF, Garry W. Turner GW, Egan JFX, Nardi DA. Use of the transverse cerebellar diameter/ abdominal circumference ratio in pregnancies at risk for intrauterine growth retardation. J Clin Ultrasound 1994;22: 497-502.

9. Chavez MR, Ananth CV, Smulian JC, Yeo L, Oyelese Y, Vintzleos AM. Fetal transcerebellar diameter measurement with particular emphasis in the third trimester: A reliable predictor of gestational age. Am J Obstet Gynaecol 2004;191:979-84. 